\title{
On the sum of the Lah numbers and zeros of the Kummer confluent hypergeometric function
}

\author{
Feng Qi \\ Institute of Mathematics, \\ Henan Polytechnic University, China; \\ College of Mathematics, \\ Inner Mongolia University for \\ Nationalities, China; \\ Department of Mathematics, \\ College of Science, \\ Tianjin Polytechnic University, China \\ email: qifeng618@gmail.com \\ qifeng618@hotmail.com \\ qifeng618@qq.com \\ https://qifeng618. wordpress.com
}

\author{
Bai-Ni Guo \\ School of Mathematics and Informatics, \\ Henan Polytechnic University, China \\ email: bai.ni.guo@gmail.com \\ bai.ni.guo@hotmail.com
}

\begin{abstract}
In the paper, the authors find the sum of the Lah numbers and make sure that the Kummer confluent hypergeometric function ${ }_{1} F_{1}(n+1 ; 2 ; z)$ has only $n-1$ real and negative zeros.
\end{abstract}

\section{Notation and main results}

In combinatorics, the Bell numbers, usually denoted by $B_{n}$ for $n \in\{0\} \cup \mathbb{N}$, count the number of ways a set with $n$ elements can be partitioned into disjoint

2010 Mathematics Subject Classification: Lah number, sum, Kummer confluent hypergeometric function, zero

Key words and phrases: Primary 11B83; Secondary 05A10, 05A19, 11B65, 11B99, 30C15, $33 \mathrm{C} 15,65 \mathrm{~B} 10$ 
and nonempty subsets. These numbers have been studied by mathematicians since the 19th century, and their roots go back to medieval Japan, but they are named after Eric Temple Bell, who wrote about them in the 1930s. Every Bell number $B_{\mathfrak{n}}$ can be generated by

$$
e^{e^{x}-1}=\sum_{k=0}^{\infty} \frac{B_{k}}{k !} x^{k}
$$

or, equivalently, by

$$
e^{e^{-x}-1}=\sum_{k=0}^{\infty}(-1)^{k} B_{k} \frac{x^{k}}{k !} .
$$

In combinatorics, the Stirling numbers arise in a variety of combinatorics problems. They are introduced in the eighteen century by James Stirling. There are two kinds of the Stirling numbers: the Stirling numbers of the first and second kinds. Every Stirling number of the second kind, usually denoted by $S(n, k)$, is the number of ways of partitioning a set of $n$ elements into $k$ nonempty subsets, can be computed by

$$
S(n, k)=\frac{1}{k !} \sum_{i=0}^{k}(-1)^{i}\left(\begin{array}{l}
k \\
i
\end{array}\right)(k-i)^{n},
$$

and can be generated by

$$
\frac{\left(e^{x}-1\right)^{k}}{k !}=\sum_{n=k}^{\infty} S(n, k) \frac{x^{n}}{n !}, \quad k \in\{0\} \cup \mathbb{N} .
$$

In combinatorics, the Lah numbers, discovered by Ivo Lah in 1955 and usually denoted by $L(n, k)$, count the number of ways a set of $n$ elements can be partitioned into $k$ nonempty linearly ordered subsets and have an explicit formula

$$
L(n, k)=\left(\begin{array}{l}
n-1 \\
k-1
\end{array}\right) \frac{n !}{k !} .
$$

The Lah numbers $L(n, k)$ can also be interpreted as coefficients expressing rising factorials $(x)_{n}$ in terms of falling factorials $\langle x\rangle_{n}$, where

$$
(x)_{n}= \begin{cases}x(x+1)(x+2) \ldots(x+n-1), & n \geq 1 \\ 1, & n=0\end{cases}
$$


and

$$
\langle x\rangle_{n}= \begin{cases}x(x-1)(x-2) \ldots(x-n+1), & n \geq 1, \\ 1, & n=0 .\end{cases}
$$

In combinatorics and the theory of polynomials, the partial Bell polynomials $B_{n, k}\left(x_{1}, x_{2}, \ldots, x_{n-k+1}\right)$ for $n \geq k \geq 0$ can be defined by

$$
B_{n, k}\left(x_{1}, x_{2}, \ldots, x_{n-k+1}\right)=\sum_{\substack{1 \leq i \leq n, \ell_{i} \in\{0\} \cup \mathbb{N} \\
\sum_{\begin{subarray}{c}{n=1 \\
\sum}=1 }} i_{i}=n} \\
{\substack{i=1 \\
\ell_{i}=k}}\end{subarray}} \frac{n !}{\prod_{i=1}^{n-k+1} \ell_{i} !} \prod_{i=1}^{n-k+1}\left(\frac{x_{i}}{i !}\right)^{\ell_{i}}
$$

and satisfy

$$
B_{n, k}(1 !, 2 !, \ldots,(n-k+1) !)=L(n, k) .
$$

The complete Bell polynomials $\mathrm{Y}_{n}\left(\mathrm{x}_{1}, \mathrm{x}_{2}, \ldots, \mathrm{x}_{\mathrm{n}}\right)$ are defined [3, p. 134] by

$$
Y_{n}\left(x_{1}, x_{2}, \ldots, x_{n}\right)=\sum_{k=1}^{n} B_{n, k}\left(x_{1}, x_{2}, \ldots, x_{n-k+1}\right)
$$

and

$$
Y_{0}\left(x_{1}, x_{2}, \ldots, x_{n}\right)=1
$$

In the theory of special functions, the generalized hypergeometric series

$$
{ }_{p} F_{q}\left(a_{1}, \ldots, a_{p} ; b_{1}, \ldots, b_{q} ; z\right)=\sum_{n=0}^{\infty} \frac{\left(a_{1}\right)_{n} \cdots\left(a_{p}\right)_{n}}{\left(b_{1}\right)_{n} \cdots\left(b_{q}\right)_{n}} \frac{z^{n}}{n !}
$$

is defined for complex numbers $a_{i} \in \mathbb{C}$ and $b_{i} \in \mathbb{C} \backslash\{0,-1,-2, \ldots\}$ and for positive integers $p, q \in \mathbb{N}$. The generalized hypergeometric series ${ }_{p} F_{q}\left(a_{1}, \ldots, a_{p}\right.$; $\left.b_{1}, \ldots, b_{q} ; z\right)$ converges absolutely for all $z \in \mathbb{C}$ if $p \leq q$, for $|z|<1$ if $p=q+1$, and for $|z|=1$ if $p=q+1$ and $\mathfrak{R}\left[b_{1}+\cdots+b_{q}-\left(a_{1}+\cdots+a_{p}\right)\right]>0$. Specially, the series

$$
{ }_{1} F_{1}(a ; b ; z)=\sum_{k=0}^{\infty} \frac{(a)_{k}}{(b)_{k}} \frac{z^{k}}{k !}
$$

is called the Kummer confluent hypergeometric function and it is analytic for all $z \in \mathbb{C}$. See $[4$, pp. 3-5].

In [5] and [7], two explicit formulas for the Bell numbers $B_{n}$ in terms of the Stirling numbers of the second kind $S(n, k)$ together with the Kummer confluent hypergeometric function ${ }_{1} F_{1}(k+1 ; 2 ; 1)$ and the Lah numbers $L(n, k)$ 
respectively were established as follows. For $n \in \mathbb{N}$, the Bell numbers $B_{n}$ can be expressed as

$$
B_{n}=\frac{1}{e} \sum_{k=1}^{n}(-1)^{n-k}{ }_{1} F_{1}(k+1 ; 2 ; 1) k ! S(n, k)
$$

and

$$
B_{n}=\sum_{k=1}^{n}(-1)^{n-k}\left[\sum_{\ell=1}^{k} L(k, \ell)\right] S(n, k) .
$$

Comparing the formulas (4) with (5) motivates us to conjecture that

$$
\frac{k !}{e}{ }_{1} F_{1}(k+1 ; 2 ; 1)=\sum_{\ell=1}^{k} L(k, \ell), \quad k \in \mathbb{N} .
$$

With the help of the famous software Mathematica 9, we can verify that the equality (6) holds true for $1 \leq k \leq 9$ and they equal the following values respectively:

$$
\text { e, } \frac{3}{2} e, \quad \frac{13}{6} e, \quad \frac{73}{24} e, \quad \frac{167}{40} e, \quad \frac{4051}{720} e, \quad \frac{37633}{5040} e, \frac{43817}{4480} e, \frac{4596553}{362880} e .
$$

This hints us that the above conjecture is true.

The aim of this paper is to prove a more general conclusions than the above conjecture. This general conclusion can be restated as the following theorems.

Theorem 1 For $z \in \mathbb{C}$ and $\mathrm{n} \in \mathbb{N}$, the formula

$$
\sum_{k=1}^{n} L(n, k) z^{k-1}=\frac{n !}{e^{z}}{ }_{1} F_{1}(n+1 ; 2 ; z)
$$

is true. Specially, for $\mathrm{n} \in \mathbb{N}$, the Lah number $\mathrm{L}(\mathrm{n}, \mathrm{k})$ and the complete Bell polynomials $\mathrm{Y}_{\mathfrak{n}}\left(\mathrm{x}_{1}, \mathrm{x}_{2}, \ldots, \mathrm{x}_{\mathfrak{n}}\right)$ satisfy

$$
\sum_{k=1}^{n} L(n, k)=\frac{n !}{e}{ }_{1} F_{1}(n+1 ; 2 ; 1)
$$

and

$$
Y_{n}(1 !, 2 !, \ldots, n !)=\frac{n !}{e}{ }_{1} F_{1}(n+1 ; 2 ; 1)
$$

respectively. 
Theorem 2 The Kummer confluent hypergeometric function ${ }_{1} \mathrm{~F}_{1}(\mathrm{n}+1 ; 2 ; z)$ has only $\mathrm{n}-1$ real and negative zeros.

Remark 1 The equations in (4) can be rewritten as

$$
\sum_{k=1}^{n}(-1)^{n-k} a_{k} S(n, k)=B_{n}
$$

where $\mathrm{a}_{\mathrm{k}}$ is sequence A000262 in the Online Encyclopedia of Integer Sequences. Such a sequence $\mathrm{a}_{\mathrm{k}}$ has a nice combinatorial interpretation: it counts "the sets of lists, or the number of partitions of $\{1,2 \ldots, \mathrm{k}\}$ into any number of lists, where a list means an ordered subset." This reveals the combinatorial interpretation of the special sequence $\mathrm{k}_{1} \mathrm{~F}_{1}(\mathrm{k}+1 ; 2 ; 1)$ and the total sum $\mathcal{L}_{\mathrm{k}}=$ $\sum_{\ell=1}^{k} \mathrm{~L}(k, \ell)$ of the Lah numbers $\mathrm{L}(\mathrm{k}, \ell)$.

\section{Proofs of theorems}

We now start out to prove Theorems 1 and 2.

Proof. [Proof of Theorem 1] It is easy to see that the equality (9) follows from substituting (1) into (8) and making use of (2) and (3). Hence, in what follows, we pay our attention to the proof of the formula (7).

In $[6$, p. 79 , Theorem 2.1], we obtained

$$
\sum_{k=1}^{n} L(n, k) x^{k}=\frac{e^{-x}}{x^{n}} \int_{0}^{\infty} I_{1}(2 \sqrt{t}) t^{n-1 / 2} e^{-t / x} d t
$$

for $n \in \mathbb{N}$ and $x>0$, where the modified Bessel function of the first kind $I_{v}(z)$ can be defined by

$$
\mathrm{I}_{v}(z)=\sum_{\mathrm{k}=0}^{\infty} \frac{1}{\mathrm{k} ! \Gamma(v+k+1)}\left(\frac{z}{2}\right)^{2 \mathrm{k}+v}
$$

for $v \in \mathbb{R}$ and $z \in \mathbb{C}$. See $[1$, p. 375, 9.6.10]. Substituting (11) for $v=1$ 
into (10) and straightforward computing arrive at

$$
\begin{aligned}
\sum_{k=1}^{n} L(n, k) x^{k} & =\frac{e^{-x}}{x^{n}} \int_{0}^{\infty} \sum_{k=0}^{\infty} \frac{1}{k !(k+1) !} t^{n+k} e^{-t / x} d t \\
& =\frac{e^{-x}}{x^{n}} \sum_{k=0}^{\infty} \frac{1}{k !(k+1) !} \int_{0}^{\infty} t^{n+k} e^{-t / x} d t \\
& =\frac{e^{-x}}{x^{n}} \sum_{k=0}^{\infty} \frac{1}{k !(k+1) !} x^{n+k+1} \Gamma(n+k+1) \\
& =e^{-x} \sum_{k=0}^{\infty} \frac{(n+k) !}{k !(k+1) !} x^{k+1} \\
& =n ! x e^{-x} \sum_{k=0}^{\infty} \frac{(n+1)_{k}}{(2)_{k}} \frac{x^{k}}{k !} \\
& =n ! x e^{-x}{ }_{1} F_{1}(n+1 ; 2 ; x) .
\end{aligned}
$$

Therefore, it follows that

$$
\sum_{k=1}^{n} L(n, k) x^{k-1}=\frac{n !}{e^{x}}{ }_{1} F_{1}(n+1 ; 2 ; x)
$$

for $x>0$ and $n \in \mathbb{N}$.

Since the functions

$$
\sum_{k=1}^{n} L(n, k) z^{k-1} \text { and } \frac{n !}{e^{z}}{ }_{1} F_{1}(n+1 ; 2 ; z)
$$

are entire functions, that is, they are analytic on the whole complex plane $\mathbb{C}$, by the uniqueness theorem of analytic functions in the theory of complex functions, see [17, p. 210, Corollary], and by the formula (12), we easily derive the formula (7) for $z \in \mathbb{C}$ and $n \in \mathbb{N}$. The proof of Theorem 1 is complete. Proof. [Proof of Theorem 2] In [2, Lemma], the authors stated that if

$$
\mathrm{P}_{\mathrm{m}, \mathrm{k}}(\mathrm{x})=\sum_{\mathrm{n}=1}^{\mathrm{m}} \mathrm{L}_{\mathrm{k}}(\mathrm{m}, \mathrm{n}) \mathrm{x}^{\mathrm{n}},
$$

then the $m$ roots of $P_{m, k}(x)$ are real, distinct, and non-positive for all $m \in \mathbb{N}$, where the associated Lah numbers $L_{k}(m, n)$ for $k>0$ can be defined by

$$
L_{k}(m, n)=\frac{m !}{n !} \sum_{r=1}^{n}(-1)^{n-r}\left(\begin{array}{l}
n \\
r
\end{array}\right)\left(\begin{array}{c}
m+r k-1 \\
m
\end{array}\right)
$$


and $L_{k}(m, n)=0$ for $n>m$. Since $L_{1}(m, n)=L(m, n)$, see [2, p. 158, Eq. (4)], when $k=1$, the polynomial $P_{m, k}(x)$ becomes

$$
P_{m, 1}(x)=\sum_{n=1}^{m} L(m, n) x^{n} .
$$

The formula (7) implies that the integer polynomial $\frac{P_{m, 1}(x)}{x}$ have the same zeros as the Kummer confluent hypergeometric function ${ }_{1}{ }_{F_{1}}(n+1 ; 2 ; z)$. Since the Kummer confluent hypergeometric function ${ }_{1} F_{1}(n+1 ; 2 ; z)$ has no positive zero, the zeros of $P_{m, 1}(x)$ are non-positive, and then the Kummer confluent hypergeometric function ${ }_{1} F_{1}(n+1 ; 2 ; z)$ has only $n-1$ real and negative zeros. The proof of Theorem 2 is complete.

Remark 2 The formula (5) has been generalized by R. B. Corcinoy, J. T. Malusay, J. A. Cillar, G. J. Rama, O. V. Silang, and I. M. Tacoloy in Philippines. There are more new results in [12] and [13, Section 5] for the Bell numbers $\mathrm{B}_{\mathrm{n}}$.

Remark 3 There are some new and closely related results published in [9, 10, 11, 14, 15, 16, 18] and references cited therein.

Remark 4 This paper is a revised version of the preprint [8].

\section{References}

[1] M. Abramowitz and I. A. Stegun (Eds), Handbook of Mathematical Functions with Formulas, Graphs, and Mathematical Tables, National Bureau of Standards, Applied Mathematics Series 55, 10th printing, Dover Publications, New York and Washington, 1972.

[2] J. C. Ahuja and E. A. Enneking, Concavity property and a recurrence relation for associated Lah numbers, Fibonacci Quart., 17 (2) (1979), $158-161$.

[3] L. Comtet, Advanced Combinatorics: The Art of Finite and Infinite Expansions, Revised and Enlarged Edition, D. Reidel Publishing Co., Dordrecht and Boston, 1974.

[4] G. Gasper and M. Rahman, Basic Hypergeometric Series, With a foreword by Richard Askey, Second edition, Encyclopedia of Mathematics 
and its Applications, 96, Cambridge University Press, Cambridge, 2004; Available online at http://dx.doi.org/10.1017/CB09780511526251.

[5] B.-N. Guo and F. Qi, An explicit formula for Bell numbers in terms of Stirling numbers and hypergeometric functions, Glob. J. Math. Anal., 2 (4) (2014), 243-248; Available online at http://dx.doi.org/10.14419/ gjma.v2i4.3310.

[6] B.-N. Guo and F. Qi, Some integral representations and properties of Lah numbers, J. Algebra Number Theory Acad., 4 (3) (2014), 77-87.

[7] F. Qi, An explicit formula for the Bell numbers in terms of the Lah and Stirling numbers, Mediterr. J. Math., 13 (5) (2016), 2795-2800; Available online at http://dx.doi.org/10.1007/s00009-015-0655-7.

[8] F. Qi, On sum of the Lah numbers and zeros of the Kummer confluent hypergeometric function, ResearchGate Research (2015), available online at http://dx.doi.org/10.13140/RG.2.1.4089.9683.

[9] F. Qi, Six proofs for an identity of the Lah numbers, Online J. Anal. Comb., 10 (2015), 5 pages.

[10] F. Qi, Derivatives of tangent function and tangent numbers, Appl. Math. Comput., 268 (2015), 844-858; Available online at http://dx.doi.org/ $10.1016 / \mathrm{j}$. amc.2015.06.123.

[11] F. Qi, Diagonal recurrence relations for the Stirling numbers of the first kind, Contrib. Discrete Math. 11 (1) (2016), 22-30; Available online at http://hdl. handle.net/10515/sy5wh2dx6 and http://dx.doi . org/10515/sy5wh2dx6.

[12] F. Qi, Some inequalities for the Bell numbers, Proc. Indian Acad. Sci. 127 (4) (2017), 551-564; Available online at https://doi.org/10.1007/s12044017-0355-2.

[13] F. Qi, X.-T. Shi, and F.-F. Liu, Expansions of the exponential and the logarithm of power series and applications, Arabian J. Math., 6 (2) (2017), 95-108; Available online at https://doi.org/10.1007/s40065-017-0166-4.

[14] F. Qi, X.-T. Shi, and F.-F. Liu, Several identities involving the falling and rising factorials and the Cauchy, Lah, and Stirling numbers, Acta Univ. Sapientiae Math., 8 (2) (2016), 282-297; Available online at http: //dx.doi.org/10.1515/ausm-2016-0019. 
[15] F. Qi and X.-J. Zhang, An integral representation, some inequalities, and complete monotonicity of the Bernoulli numbers of the second kind, Bull. Korean Math. Soc., 52 (3) (2015), 987-998; Available online at http: //dx.doi.org/10.4134/BKMS.2015.52.3.987.

[16] F. Qi and M.-M. Zheng, Explicit expressions for a family of the Bell polynomials and applications, Appl. Math. Comput., 258 (2015), 597-607; Available online at http://dx.doi.org/10.1016/j.amc.2015.02.027.

[17] W. Rudin, Real and Complex Analysis, International Student Edition, McGraw-Hill Book Company, 1970.

[18] C.-F. Wei and F. Qi, Several closed expressions for the Euler numbers, J. Inequal. Appl., 2015, 2015:219, 8 pages; Available online at http: //dx.doi.org/10.1186/s13660-015-0738-9.

Received: June 17, 2017 